\title{
Field Asymmetry and Thrust Control in the GDM Fusion Propulsion System
}

\author{
Ricky Tang, ${ }^{1}$ Terry Kammash ${ }^{2}$ and Alec D. Gallimore ${ }^{3}$ \\ University of Michigan, Ann Arbor, MI, 48109
}

\begin{abstract}
[Abstract] One of the notable attributes of the Gasdynamic Mirror (GDM) fusion propulsion system is the fact that it lends itself readily to thrust control due to asymmetry in the magnetic field configuration. GDM is a magnetic plasma confinement device that has been proposed as a potential propulsion system. It differs from the standard "collisionless" mirror in that it operates at a much higher plasma density and is generally designed with a large aspect ratio to provide plasma stability. When a plasma is injected into such a device, the electrons tend to escape through the mirrors more readily due to their small mass, leaving behind an excess of ions and correspondingly a positive electrostatic potential. Such a potential accelerates the ions while slowing down the electrons until both species drift outward at equal rate. Of special importance to its value as a propulsion device is the effect of magnetic field asymmetry on such an electrostatic potential. Numerical simulations have been carried out to study this phenomenon and to quantify the role of asymmetry on the overall performance of the system. Experiments will be conducted in order to validate the theoretical and simulation models and to provide a basis for further assessment of GDM as a propulsion device with variable thrust capability.
\end{abstract}

\section{Nomenclature}

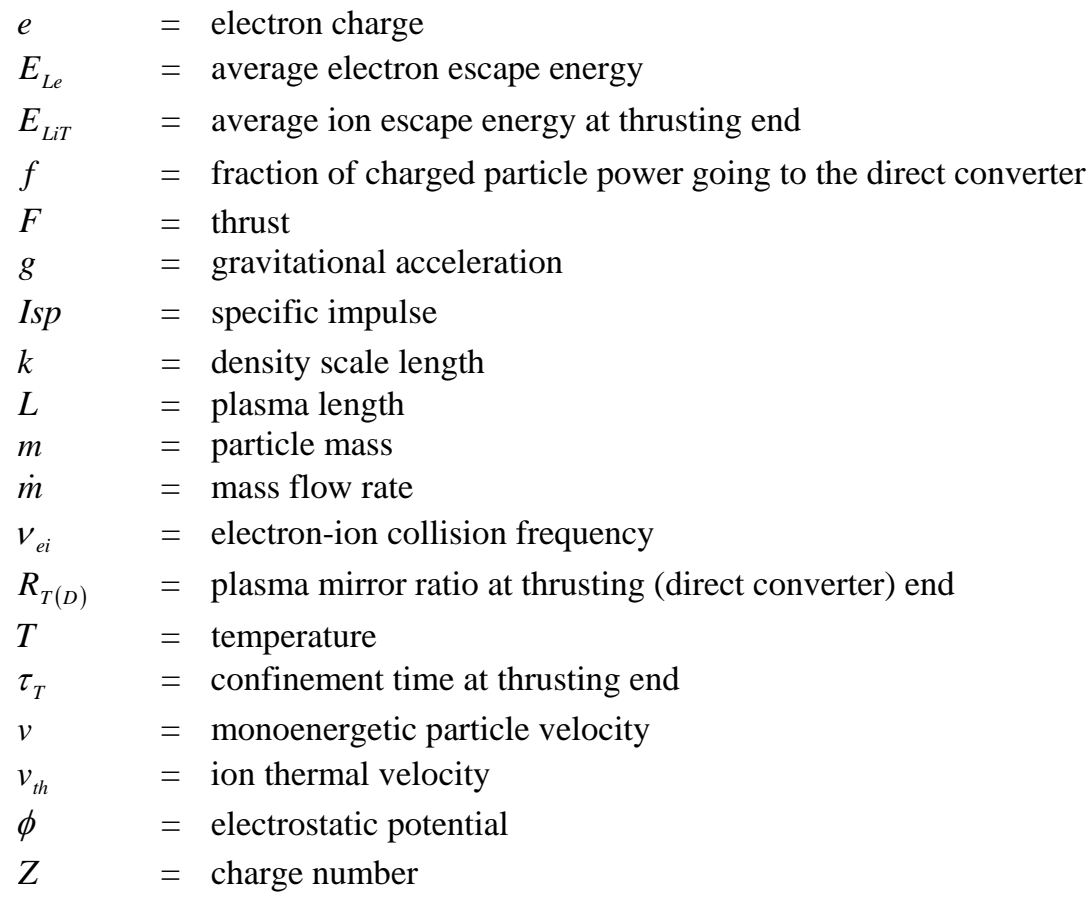

\footnotetext{
${ }^{1}$ Graduate Student, Dept. of Aerospace Engineering, 1320 Beal Ave., Ann Arbor, MI 48109.

${ }^{2}$ Professor Emeritus, Dept. of Nuclear Engineering and Radiological Sciences, 2355 Bonisteel Blvd., Ann Arbor, MI 48109, AIAA Associate Fellow.

${ }^{3}$ Professor, Dept. of Aerospace Engineering, 1320 Beal Ave., Ann Arbor, MI 48109, AIAA Associate Fellow.
} 


\section{Introduction}

$\mathrm{T}$ HE gasdynamic mirror (GDM) propulsion system is a magnetic mirror confinement system in which the propellant (in the form of a dense plasma) is confined for a period of time before being accelerated through the magnetic nozzle to produce thrust. Magnetic field asymmetry is necessary to bias the ion escape to the thrusting end of the GDM, while a direct converter on the opposite non-thrusting end recovers some of the energy that would otherwise be lost due to the escaping ions. In order to achieve better confinement such that the propellant can be heated to the desired temperature and to provide plasma stability leading to more efficient utilization of the confining magnetic field, the system is designed with a large aspect ratio (i.e. length >> plasma radius). For such a system, the magnetic configuration is effectively that of a meridional nozzle, where the fluid flow velocity is everywhere parallel to the magnetic field lines. Unlike a 'collisionless' mirror system, the requirement of a high density plasma inside the GDM ensures that the ion-ion collision mean free path is much smaller than a characteristic dimension of the system, e.g. its length, which underlies the confinement principle of the GDM. Under these conditions, the 'collisional' plasma behaves much like a continuous medium (a fluid), such that its escape from the system is analogous to the flow of a gas into vacuum from a vessel with a hole, and the system can therefore be analyzed as such.

We have previously ${ }^{1}$ assessed the GDM by solving the appropriate particle and energy balance equations in order to establish the physical properties of the system and its propulsive capabilities. However, Ref. 1 did not address the electrostatic potential that arises due to the initial rapid loss of the electrons. This self-generated electric field significantly alters the particle dynamics, which was examined in Ref. 2, along with its effect on the escape energies of the electrons and ions (hence the thrust and specific impulse of the system) for a symmetric magnetic field configuration. As mentioned above, however, an asymmetric magnetic field configuration is desired from the standpoint of a propulsion system, and Ref. 3 addressed this by allowing different mirror ratios (ratio of magnetic field at the mirror to that at the center) at the two ends of the GDM. Increasing the mirror ratio (i.e. increasing the mirror magnetic field strength) therefore reduces plasma flow.

\section{Analytical Examination of the Effect of Field Asymmetry}

The goal of this paper is to investigate how field asymmetry affects the propulsive capabilities of the GDM system. The thrust and specific impulse of the system are given by the followings.

$$
\begin{gathered}
F=\dot{m}_{i} v_{i}+\dot{m}_{e} v_{e} \\
I s p=\frac{m_{i} v_{i}+m_{e} v_{e}}{g\left(m_{i}+m_{e}\right)}
\end{gathered}
$$

where $v_{i}$ and $v_{e}$ are respectively the average escape velocities of the ions and electrons and are proportional to $\sqrt{E_{L i T}+e \phi}$ and $\sqrt{E_{L e}-e \phi}$. On the other hand, both the ion and electron mass flow rates are proportional to $1 / \tau_{T}$.

Consequently, we see that field asymmetry directly influences the thrust and Isp of the GDM through the particle escape energies, confinement time, and the ambipolar potential itself. The ambipolar potential for an asymmetric GDM was derived in our previous analyses; ${ }^{3}$ it is obtained by solving Eq. (3a) iteratively.

$$
\begin{aligned}
\left(1-\frac{2}{3} x^{2}\right)[1-e r f(x)] & +\frac{2}{\sqrt{\pi}} x \exp \left(-x^{2}\right)=\frac{\delta_{T}+\delta_{D}}{2 T_{e}}+\frac{2}{3} \frac{m_{e}}{m_{i}} Z^{2} x^{2} \\
\delta_{j} & \equiv \frac{L}{4 R_{j} k} m_{e} v_{e i}\left(\frac{8 T_{i}}{\pi m_{i}}\right)^{1 / 2} \\
x & \equiv \sqrt{\frac{3 e \phi}{2 T_{e}}}
\end{aligned}
$$

Once the potential is known, it can be used to evaluate the electron and ion escape energies, given by the following expressions. ${ }^{3}$ 


$$
\begin{gathered}
E_{L e}=\frac{\left(5-2 x^{2}\right)[1-\operatorname{erf}(x)]+\frac{2}{\sqrt{\pi}}\left(5+\frac{4}{3} x^{2}\right) x \exp \left(-x^{2}\right)}{2\left(1-\frac{2}{3} x^{2}\right)[1-\operatorname{erf}(x)]+\frac{4}{\sqrt{\pi}} x \exp \left(-x^{2}\right)} T_{e} \\
E_{L i T}=\frac{2+\frac{m_{e}}{m_{i}} Z^{2} \frac{T_{e}}{\delta_{T}} x^{2}}{1+\frac{2}{3} \frac{m_{e}}{m_{i}} Z^{2} \frac{T_{e}}{\delta_{T}} x^{2}} T_{i}
\end{gathered}
$$

We recall that Eqs. (4) and (5) give the average energies of escaping electrons and ions as they leave the plasma chamber, the ambipolar potential must be added to (subtracted from) the ion (electron) energy to obtain their energies outside the chamber, as alluded to earlier. It is clear from Eqs. (4) and (5) that both have a direct and complicated dependence on the potential, which in turn varies with the mirror ratios. Furthermore, the ion energy has an explicit dependence on the mirror ratio at the thrusting end of the GDM. On the other hand, the mirror ratio does not directly influence the electron energy; it affects the electrons only indirectly through the potential. This is because they have such small mass and high velocity that they essentially do not see the mirrors.

Finally, the loss rate or confinement time $\tau_{T}$ depends explicitly on the ambipolar potential and the mirror ratio according to the following expression. ${ }^{3}$

$$
\tau_{T}=\frac{R_{T} L}{v_{t h}\left[1+\left(m_{e} / m_{i}\right) Z^{2}\left(e \phi / \delta_{T}\right)\right]}
$$

From this brief qualitative analysis, we can see that the end result of varying the magnetic field strength at the mirrors and/or the central region, such that the mirror ratios change, is that the thrust and the Isp of the system change accordingly.

\section{Numerical Simulation}

\section{A. Results on Varying the Mirror Ratios at a Fixed Plasma Temperature and Density}

Due to the interdependence of the different variables, such as the potential, plasma length, and mirror ratio, and the coupling of the various equations governing each variable, in addition to conservation equations, such as particle and energy conservation, that must be considered, it is difficult to assess the full functional dependency analytically. A computer code was written to model the physics inside the GDM. The code utilizes all of the above equations, plus others that weren't shown here, to solve for self-consistent values for the various quantities.

Fig. 1 shows how the ambipolar potential varies with the mirror ratio $R_{T}$ at the thrusting end, at a given plasma temperature and density $(10 \mathrm{keV}$ and $5 \times 10^{17} \mathrm{~cm}^{-3}$ ), for various mirror ratio $R_{D}$ at the direct converter end. The GDM was simulated as a steady-state deuterium-tritium (DT) fusion

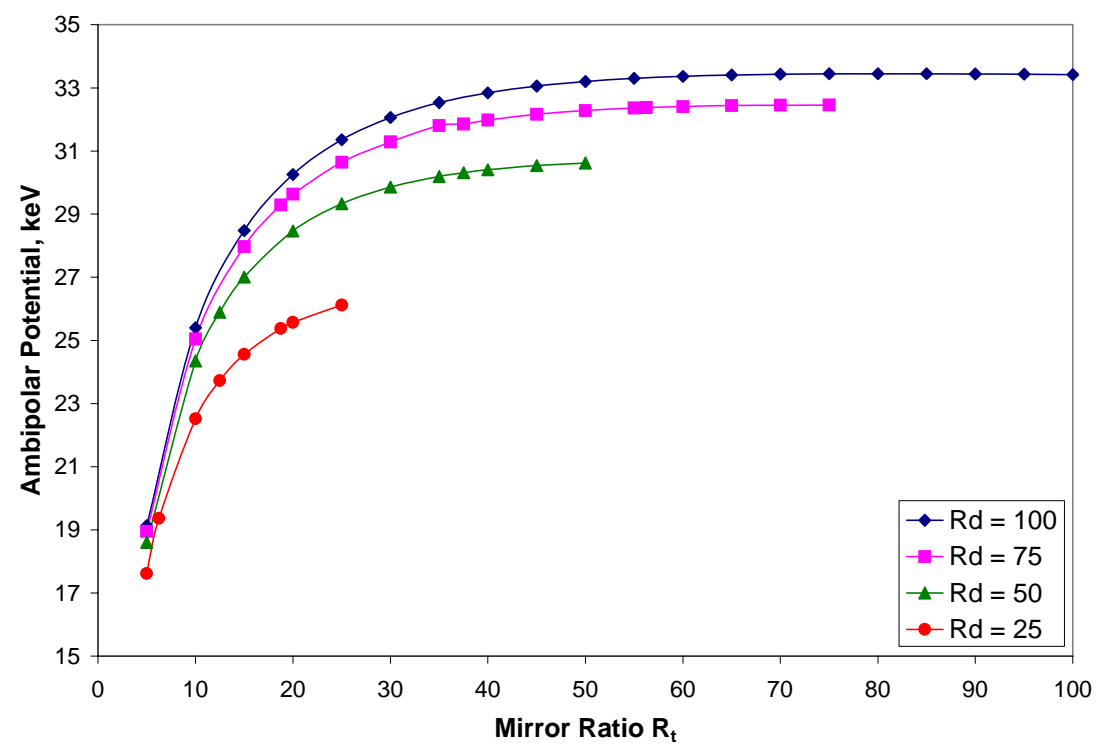

Figure 1. Ambipolar potential as a function of the two mirror ratios $R_{T}$ and $R_{D}$ at $10 \mathrm{keV}$ temperature and a density of $5 \times 10^{17} \mathrm{~cm}^{-3}$.

American Institute of Aeronautics and Astronautics 
system. We can see that for a given $R_{D}$, the potential increases approximately logarithmically with $R_{T}$, and at a given $R_{T}$, it increases with increasing $R_{D}$. Each line in the plot ends when $R_{T}=R_{D}$, where half of the charged particle power appears as thrust power and the other half goes to the direct converter with a certain efficiency ( $80 \%$ in these simulations). There is no merit for $R_{T}>R_{D}$ in a propulsion system since the thrust power would be less than $50 \%$ in that case, and most of the power would then go through the direct converter leading to greater loss. Finally, we note that the ambipolar potential is quite significant; in fact for the settings we used, the potential is about the same or greater than the ion escape energy, as seen in Fig. 2. This significantly enhances the energy (velocity) of the ions, which provide the bulk of the thrust, as they leave the GDM chamber.

Fig. 2 shows the behavior of the average electron escape energy $E_{L e}$ and the average ion escape energy $E_{L i T}$ at the thrusting end. The electron energy has the same general dependence on $R_{T}$ as the ambipolar potential. This is consistent with the observation earlier that due to their small mass and high energy, the electrons do not see the mirror and are not directly affected by it, but are only influenced via the ambipolar potential. On the other hand, the ions are directly affected by the mirror, as well as the potential, which is evidenced in Fig. 2.

Fig. 3 shows the dependence of the ambipolar confinement time $\tau_{T}$ at the thrusting end. Again, it increases in an approximately logarithmic fashion with $R_{T}$ for a given $R_{D}$. For small $R_{T}$, it increases with increasing $R_{D}$, whereas the trend flips as $R_{T}$ increases. However,

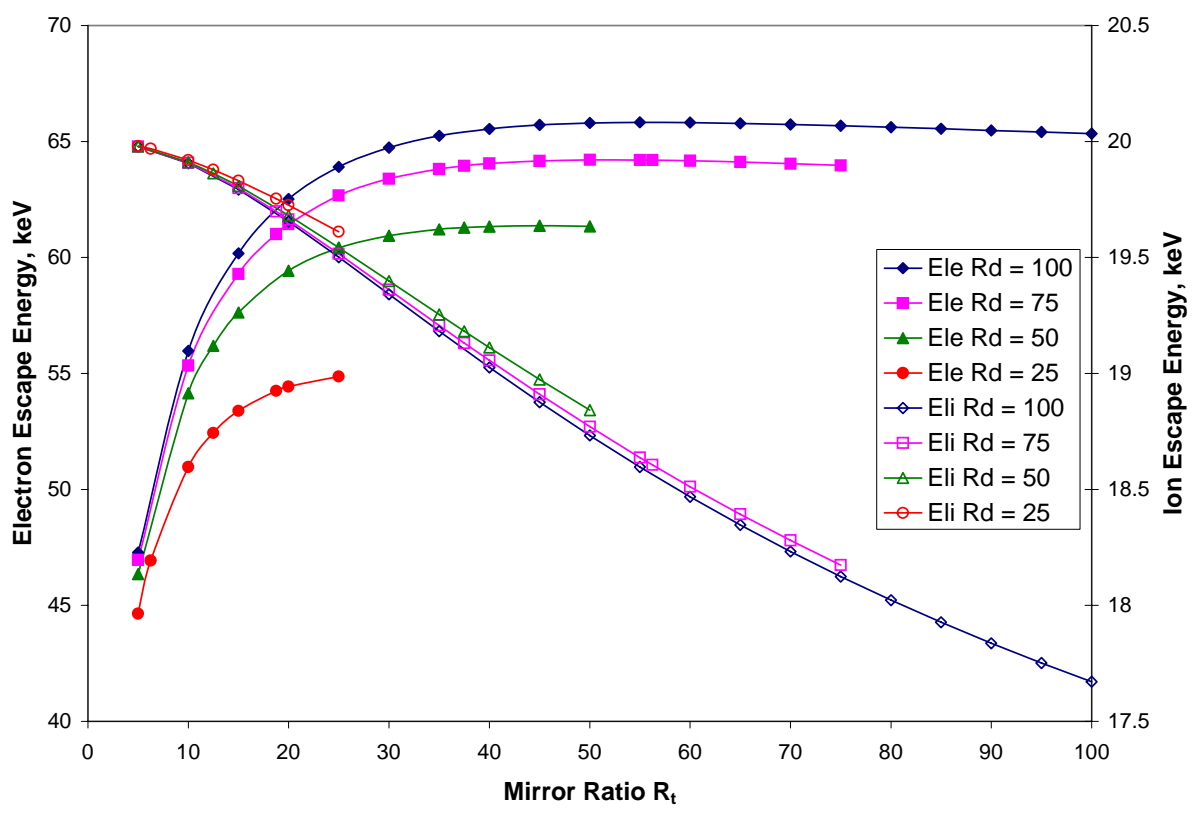

Figure 2. Average electron ( $\left.E_{L e}\right)$ and ion ( $E_{L i T}$ ) escape energies as a function of the mirror ratios, under the same simulation conditions as Fig. 1.

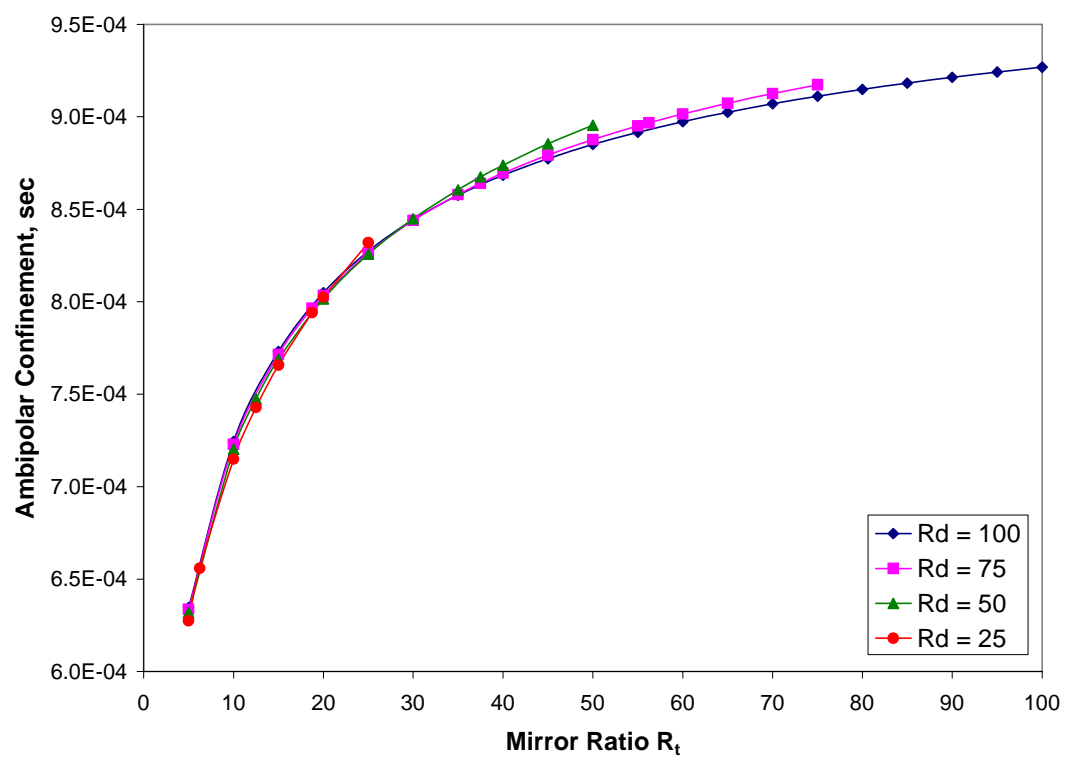

Figure 3. Ambipolar confinement time at the thrusting end as a function of the mirror ratios, under the same conditions as Fig. 1. 
the overall dependence on $R_{D}$ is relatively weak.

Figs. 4 and 5 depict respectively how the thrust and Isp of the GDM vary with $R_{T}$ and $R_{D}$. We can see that the thrust decreases approximately exponentially with $R_{T}$ for a given $R_{D}$; this behavior is due to its dependence on the ambipolar confinement time that enters into the mass flow rate calculation. Its dependence on $R_{D}$, however, is not significant, even though closer inspection suggests that for a given $R_{T}$, the thrust decreases slightly with decreasing $R_{D}$. We recall that the average escape velocities of the ions and electrons are proportional to $\sqrt{E_{L i T}+e \phi}$ and $\sqrt{E_{L e}-e \phi}$, respectively, and from Figs. 1 and 2 we can deduce that both of these quantities increase with increasing $R_{D}$, consistent with the thrust results. In addition, for a given $R_{D}$, the particle velocity increases, reaches a maximum, and then gently decreases with increasing $R_{T}$. This behavior is captured by the Isp results in Fig. 5.

Finally, Fig. 6 relates the values of the two mirror ratios $R_{T}$ and $R_{D}$ to the fraction $f$ of charged particle power that goes to the direct converter.

\section{B. Results on Varying Plasma Density}

The previous section examines how the various quantities are affected by the mirror ratios for a given plasma temperature and density. In this section, we will briefly look at how the GDM propulsive capabilities depend on the plasma number density, for a given $R_{D}$, which is arbitrarily set at 100 . We looked at two different densities, $n=10^{16} \mathrm{~cm}^{-3}$ and $n=5 \times 10^{17} \mathrm{~cm}^{-3}$. Fig. 7 shows the results for the ambipolar potential. We can see that at very small values of $R_{T}$, the potential is essentially independent of the density. However, as $R_{T}$ increases, the potential for the lower density starts to have slightly higher values. Fig. 8 shows the thrust and Isp. As before, the Isp follows 


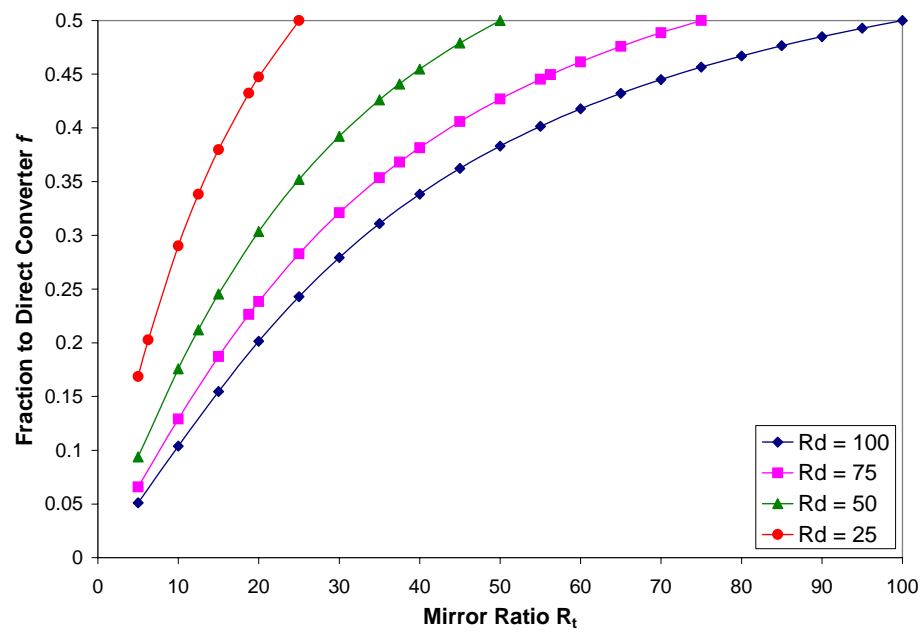

Figure 6. Fraction of charged particle power going to the direct converter, under the same conditions as Fig. 1.

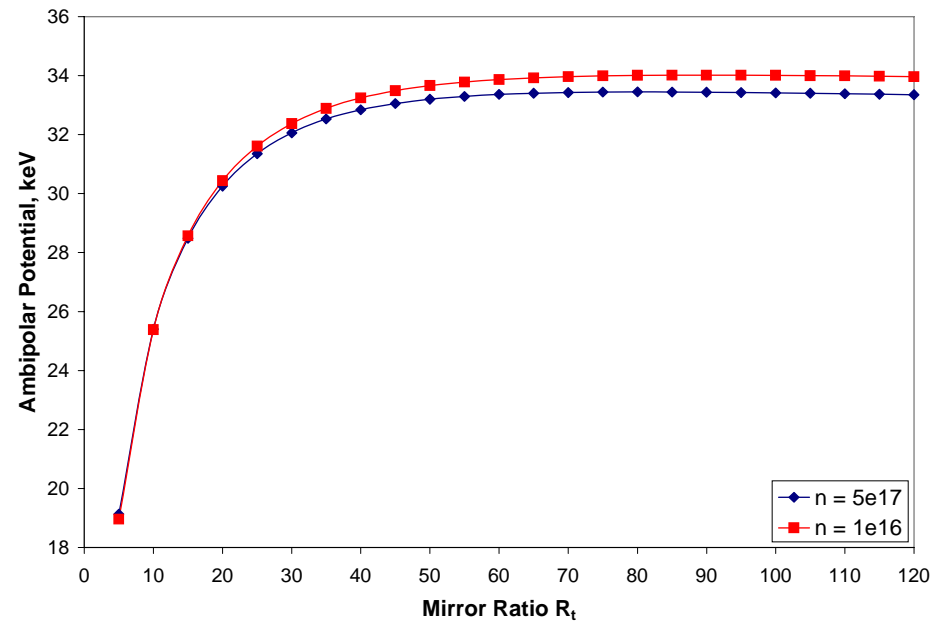

Figure 7. Ambipolar potential as a function of $R_{T}$ for $R_{D}=100$ at $10 \mathrm{keV}$ temperature and two different densities.

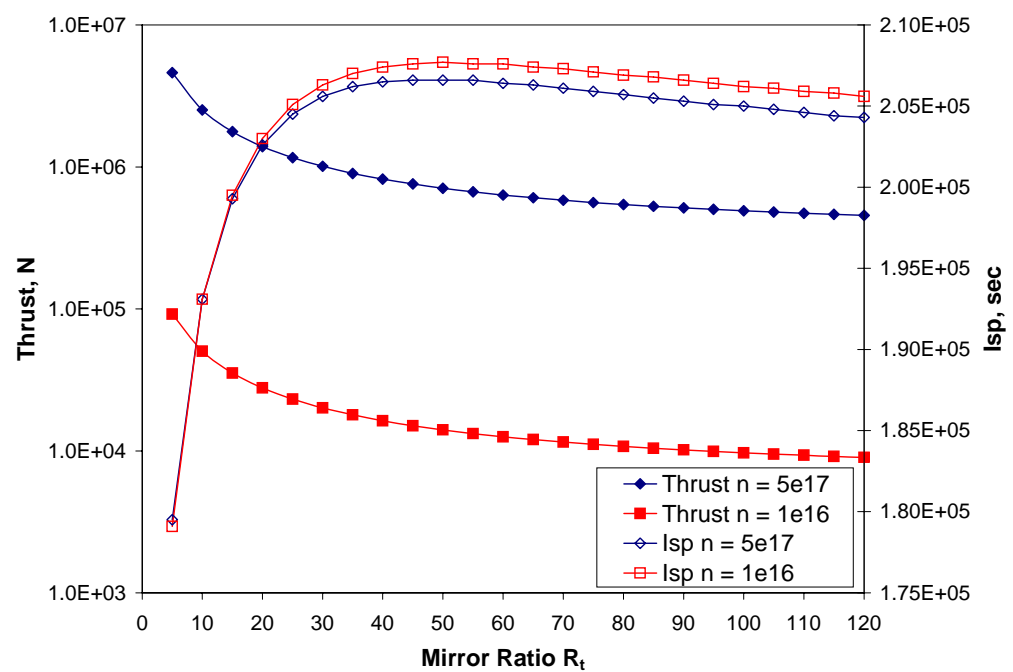

Figure 8. Thrust and Isp results under the same simulation conditions as Fig. 7.

American Institute of Aeronautics and Astronautics 
the trend of the potential, and the thrust shows an approximately exponential decrease. In addition, the thrust is about 50 times smaller everywhere for the lower density, since the mass flow rate (and hence the thrust) is directly proportional to the number density, which is now 50 times smaller. Consequently, we see that changing the number density has no effect on the overall behavior, even though the absolute magnitude may be different.

\section{Effects of Different $\boldsymbol{R}_{T}$ and $\boldsymbol{R}_{D}$ combinations for a Given $\boldsymbol{R}_{T} / \boldsymbol{R}_{D}$ Ratio}

Finally, we would like to look at how the performance of the GDM varies for a given $R_{T} / R_{D}$ ratio. As before, the simulations were run at a $10 \mathrm{keV}$ temperature and a density of $n=5 \times 10^{17} \mathrm{~cm}^{-3}$, with the GDM running in steady-state DT fusion mode. Table 1 shows that for a given ratio, the thrust increases as the mirror ratios are reduced. This is due to the increased mass flow through the mirrors. On the other hand, the decreasing potential due to the decreasing mirror ratios lead to a reduction in the Isp. Another observation made explicit by Table 1 is that the fraction of charged particle power that is thrust power increases slightly as the mirror ratios decrease, even though the $R_{T} / R_{D}$ ratio remains constant, as long as its value is less than 1.

\section{Conclusion}

We have presented in this paper the numerical results on how magnetic field asymmetry affects the propulsive capabilities of the GDM through its effects on the ambipolar potential, confinement time and particle escape energies. The dependency is a direct consequence of the physics model we developed

Table 1. Results on varying the $R_{T} / R_{D}$ ratio.

\begin{tabular}{cccccc}
\hline $\boldsymbol{R}_{\boldsymbol{T}} / \boldsymbol{R}_{\boldsymbol{D}}$ & $\boldsymbol{R}_{\boldsymbol{D}}$ & $\boldsymbol{R}_{\boldsymbol{T}}$ & $\boldsymbol{f}$ & $\boldsymbol{F} \mathbf{( N )}$ & $\boldsymbol{I s p} \mathbf{( s e c )}$ \\
\hline \hline \multirow{5}{*}{0.25} & 100 & 25 & 0.24 & $1.16 \times 10^{6}$ & $2.05 \times 10^{5}$ \\
& 75 & 18.75 & 0.23 & $1.46 \times 10^{6}$ & $2.01 \times 10^{5}$ \\
& 50 & 12.5 & 0.21 & $2.04 \times 10^{6}$ & $1.94 \times 10^{5}$ \\
& 25 & 6.25 & 0.20 & $3.70 \times 10^{6}$ & $1.80 \times 10^{5}$ \\
\hline \multirow{4}{*}{0.5} & 100 & 50 & 0.38 & $7.08 \times 10^{5}$ & $2.07 \times 10^{5}$ \\
& 75 & 37.5 & 0.37 & $8.45 \times 10^{5}$ & $2.05 \times 10^{5}$ \\
& 50 & 25 & 0.35 & $1.13 \times 10^{6}$ & $2.01 \times 10^{5}$ \\
& 25 & 12.5 & 0.34 & $1.99 \times 10^{6}$ & $1.89 \times 10^{5}$ \\
\hline \multirow{4}{*}{0.75} & 100 & 75 & 0.46 & $5.62 \times 10^{5}$ & $2.06 \times 10^{5}$ \\
& 75 & 56.25 & 0.45 & $6.45 \times 10^{5}$ & $2.05 \times 10^{5}$ \\
& 50 & 37.5 & 0.44 & $8.22 \times 10^{5}$ & $2.02 \times 10^{5}$ \\
& 25 & 18.75 & 0.43 & $1.38 \times 10^{6}$ & $1.93 \times 10^{5}$ \\
\hline \multirow{4}{*}{1} & 100 & 100 & 0.5 & $4.91 \times 10^{5}$ & $2.05 \times 10^{5}$ \\
& 75 & 75 & 0.5 & $5.48 \times 10^{5}$ & $2.04 \times 10^{5}$ \\
& 50 & 50 & 0.5 & $6.71 \times 10^{5}$ & $2.02 \times 10^{5}$ \\
& 25 & 25 & 0.5 & $1.08 \times 10^{6}$ & $1.94 \times 10^{5}$ \\
\hline
\end{tabular}
in earlier works. Although the simulations were done for a specific plasma temperature and density combination, we saw briefly in Section IIIB that varying the plasma conditions only affects the absolute magnitude of the various quantities and does not change the overall trend. Similarly, the absolute magnitudes of the various quantities such as thrust and Isp are inconsequential in our current study; what we are looking for is the overall trend on how these quantities are affected by the changing mirror ratios. Our study suggests the possibility that the thrust and Isp can be controlled by varying the magnetic field strengths at the mirror and/or at the central region so long as the overall mirror ratio is changed, since the various quantities depend on the mirror ratio and not on the absolute magnitude of the magnetic field. Although the simulations were carried out for a steady-state DT fusion system, the GDM can also be operated as a plasma thruster driven by an external power source. We will be conducting similar study for the GDM plasma thruster as what we have done here. Experiments will also be carried out in order to validate the theoretical and simulation models we have developed and to provide a basis for further assessment of the GDM as a propulsion system.

\section{References}

${ }^{1}$ Kammash, T., and Lee, M. J., “Gasdynamic Fusion Propulsion System for Space Exploration,” Journal of Propulsion and Power, Vol. 11, No. 3, 1995, pp. 544-553.

${ }^{2}$ Kammash, T., and Galbraith, D. L., "Improved Physics Model for the Gasdynamic Mirror Fusion Propulsion System," Journal of Propulsion and Power, Vol. 14, No. 1, 1998, pp. 24-28.

${ }^{3}$ Kammash, T., and Tang, R., "Propulsive Capability of an Asymmetric GDM Propulsion System," 42 ${ }^{\text {nd }}$ AIAA/ASME/SAE/ASEE Joint Propulsion Conference and Exhibit, Sacramento, CA, July 2006. 